\title{
Dynamic Shaping of the Defensive Peripersonal Space through Predictive Motor Mechanisms: When the "Near" Becomes "Far"
}

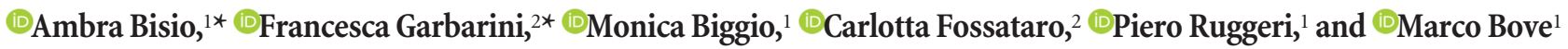 \\ ${ }^{1}$ Department of Experimental Medicine, Section of Human Physiology, University of Genoa, 16132 Genoa, Italy, and ${ }^{2}$ SpAtial, Motor and Bodily \\ Awareness-Research Group, Psychology Department, University of Turin, 10123 Turin, Italy
}

The hand blink reflex is a subcortical defensive response, known to dramatically increase when the stimulated hand is statically positioned inside the defensive peripersonal space (DPPS) of the face. Here, we tested in a group of healthy human subjects the hand blink reflex in dynamic conditions, investigating whether the direction of the hand movements (up-to/down-from the face) could modulate it. We found that, on equal hand position, the response enhancement was present only when the hand approached to (and not receded from) the DPPS of the face. This means that, when the hand is close to the face but the subject is planning to move the hand down, the predictive motor system can anticipate the consequence of the movement: the "near" becomes "far." We found similar results both in passive movement condition, when only afferent (visual and proprioceptive) information can be used to estimate the final state of the system, and in motor imagery task, when only efferent (intentional) information is available to predict the consequences of the movement. All these findings provide evidence that the DPPS is dynamically shaped by predictive mechanisms run by the motor system and based on the integration of feedforward and sensory feedback signals.

Key words: defensive peripersonal space; hand blink reflex; motor imagery; movement

Significance Statement

The defensive peripersonal space (DPPS) has a crucial role for survival, and its modulation is fundamental when we interact with the environment, as when we move our arms. Here, we focused on a defensive response, the hand blink reflex, known to increase when a static hand is stimulated inside the DPPS of the face. We tested the hand blink reflex in dynamic conditions (voluntary, passive, and imagined movements) and we found that, on equal hand position, the response enhancement was present only when the hand approached to (and not receded from) the DPPS of the face. This suggests that, through the integration of efferent and afferent signals, the safety boundary around the body is continuously shaped by the predictive motor system.

\section{Introduction}

The peripersonal space (PPS) is the space directly surrounding the body (Rizzolatti et al., 1997) within which we can act and interact. According to a recent review (de Vignemont and Iannetti, 2015), there is not a single representation of PPS but a dual model of PPS. This is based on a functional distinction between goal-directed action and bodily protection. In the present study,

Received Dec. 29, 2015; revised Dec. 15, 2016; accepted Dec. 24, 2016.

Author contributions: A.B., F.G., P.R., and M. Bove designed research; A.B., F.G., M. Biggio, and C.F. performed research; A.B., M. Biggio, C.F., and M. Bove analyzed data; A.B., F.G., M. Biggio, P.R., and M. Bove wrote the paper. The authors declare no competing financial interests.

*A.B. and F.G. equally contributed to this work.

Correspondence should be addressed to either of the following: Dr. Marco Bove, University of Genoa, Department of Experimental Medicine, Section of Human Physiology, Viale Benedetto XV 3, 16132 Genoa, Italy, E-mail: marco.bove@unige.it; or Dr. Francesca Garbarini, University of Turin, Psychology Department, SpAtial, Motor and Bodily Awareness-Research Group, Via Po 14, 10123 Turin, Italy. E-mail: fra.garbarini@gmail.com.

DOI:10.1523/JNEUROSCI.0371-16.2016

Copyright $\odot 2017$ the authors $\quad 0270-6474 / 17 / 372415-10 \$ 15.00 / 0$ we focused on the latter concept: the defensive PPS (DPPS) (Cooke and Graziano, 2003; Graziano and Cooke, 2006). The DPPS has been recently investigated in humans by recording the hand blink reflex (HBR), which is a subcortical response at the brainstem level elicited by the electrical stimulation of the median nerve at the wrist and recorded from the orbicularis oculi muscles (Sambo et al., 2012a, b; Sambo and Iannetti, 2013; Fossataro et al., 2016). In the static condition, the HBR is modulated by the hand position in space: the response dramatically increases when the stimulated hand is located close to the face, inside the DPPS.

When we interact with the surrounding environment, the modulation of the DPPS can become fundamental to prevent potentially dangerous situations. During voluntary movements, the central nervous system can estimate the final hand position using either motor outflow or sensory inflow (i.e., visual and proprioceptive inputs). These two sources of information can be combined in a predictive model, according to which, once the 
motor program is selected and sent to the periphery, an efference copy is formed to predict the future body state and the consequences of the movement, that, in turn, are compared with the actual state detected from the sensory feedback (Wolpert et al., 1995; Blakemore et al., 2002; Haggard, 2005).

Understanding the role of the predictive motor system in modulating the DPPS during movement might be a first important step toward a full comprehension of the defensive mechanisms in ecological contexts when humans move in a possible dangerous environment. To this aim, we investigated the role of predictive motor mechanisms in dynamically shaping the DPPS during upper limb voluntary movements, by recording the HBR when participants were asked to move their right forearm up toward the face (up-moving condition) or down far from the face (down-moving condition). Indeed, movements in different directions could allow us to investigate the response to a dangerous stimulus entering or leaving our DPPS. In each condition, the HBR was elicited during the forearm's movement when the amplitude of the elbow angle reached three predefined values. In turn, these three positions determined three hand distances with respect to the face (far, intermediate, and near).

We hypothesized that the predicted final consequence of the movement (either close to or far from the face) could affect the reflex response amplitude. Thus, on equal hand positions, comparing the up-moving with the down-moving condition, we expected to find a different modulation of the HBR depending on the direction of the hand movement.

During voluntary movements, intentional outflow and sensory inflow are both available to estimate the final position of the hand. Thus, to investigate the relative roles of these complementary sources of information in dynamically modulating HBR amplitude during movement, we designed two experiments, using either passive movements (where only sensory inflow is present) or motor imagery (where, on the opposite, only intentional outflow is present). In the former experiment, the subjects were asked to stay relaxed while the examiner passively moved their right arm up toward or down far from their face; in the latter, the subjects stayed still, keeping their right hand in far, intermediate, or near positions while imaging to move it up to or down from the face.

\section{Materials and Methods}

\section{Participants}

Twenty-eight participants, naive to the purpose of the experiment, were recruited for this study. They reported no previous history of neurological disorders or orthopedic problems for the right-dominant hand, as determined by the Edinburgh Handedness Inventory (Oldfield, 1971).

First, participants were tested to assess whether they showed a reproducible HBR (Sambo et al., 2012a) (see Preliminary experiment). Thirteen participants were assigned to Experiment 1, Experiment 2, and Experiment $3 \mathrm{~A}$. Ten of them $(\sim 77 \%$ of the total number, 6 females and 4 males, mean \pm SD age, $22.4 \pm 2.3$ years) showed a reproducible HBR and were thus chosen to advance to the next stages of the experimental procedure. Fifteen participants were assigned to Experiment 3B; 12 of them showed a reproducible HBR and thus continued the experimental session ( $80 \%$ of the total number, 7 females and 5 males, age, $23.1 \pm 3.3$ years). Participants gave written informed consent before taking part in the study. The study has been approved by the local ethics committee and was conducted in accordance with the Declaration of Helsinki.

\section{Experimental setup}

The HBR response was elicited by administering transcutaneous electrical stimuli to the median nerve at the right wrist, using a surface bipolar electrode attached with a velcro strap and connected to a constant current stimulator (DS7AH HV, Digitimer). As the stimulator provided constant current pulses, the trial-to-trial variability of the intensity of stimulation was negligible. Stimulus intensity was adjusted to elicit in each participant clear HBR responses (mean stimulus intensities were $27.7 \pm 9.4 \mathrm{~mA}$, range 15-42 mA). None of the participants reported painful sensations elicited by the stimulation. The stimulus duration was $200 \mu \mathrm{s}$, and the interstimulus interval was $\sim 30 \mathrm{~s}$. A twin-axis electronic goniometer (TSD130B, BIOPAC System) connected to a BIOPAC MP100 system was used to measure and record the elbow angle during movement execution. In Experiments 1 and 2, this device allowed the automatic delivery of the electrical stimulation when the elbow angle corresponded to one of the three predetermined stimulation positions.

EMG activity was recorded by means of two MP100 BIOPAC EMG channels from the orbicularis oculi muscles bilaterally, using two pairs of bipolar surface electrodes with the active electrode over the mid lower eyelid and the reference electrode laterally to the outer canthus. Signals were amplified and digitized at $1 \mathrm{kHz}$.

\section{Experimental procedure}

The experiments took place in four different sessions. In the first session, participants performed the preliminary experiment during which the HBR responses were acquired in the static condition. Participants who showed a reproducible HBR in the static condition advanced to the next stages of the study. In the second session, the selected participants executed Experiment 1 (voluntary movement). Experiment 2 (passive movement) and Experiment 3A (motor imagery from intermediate position) were randomly executed in two other different sessions. At least 1 week passed between one experimental session and the following.

Participants were seated on a comfortable chair and kept the right elbow at the limit of a table, in a position allowing the right wrist to be in front of the ipsilateral eye while moving the forearm toward the face, but never touching it. The electrical stimulation was delivered, in static condition or during voluntary and passive movements, while the participant's stimulated hand was located at three different positions relative to the face, in particular, when the elbow angle was: $10^{\circ}$ less than the maximal arm extension (far position, $\alpha 1$ ); half of the difference between the angles of maximal arm extension and flexion (intermediate position, $\alpha 2$ ); $10^{\circ}$ more than the maximal elbow flexion (near position, $\alpha 3$ ). Throughout the experiment, participants were instructed to keep their gaze on a fixation point placed at $60 \mathrm{~cm}$ from the eyes.

Preliminary experiment. Static condition. This experiment aimed to make an initial selection of those participants who showed a reproducible HBR response. These subjects were admitted to the next sessions of the study. Further, we also tested the reliability of our setup by replicating the results known in the literature. Participants were instructed, trial by trial, to put the arm in one of the three positions previously identified. After a randomly variable delay, the subject received the electrical stimulation, which was manually delivered by the experimenter. Twenty-four acquisitions were performed, 8 for each hand position. The order of the hand positions at which the participant received the electrical stimulus was pseudo-random.

Experiment 1. Voluntary movement (Fig. 1A). The aim of the present experiment was to assess whether the HBR response was modulated during voluntary movement execution and was influenced by movement direction. Participants were asked to perform two sequences of movements with the right arm: elbow flexion-extension (Block A) and elbow extension-flexion (Block B). These two blocks were introduced to avoid that participants could predict the instant of the stimulation, and the order of blocks execution was balanced across participants. In both blocks, the electrical stimulation was delivered in each trial (flexionextension or extension-flexion movements) during either elbow flexion (afterward, called up-moving condition) or elbow extension (afterward, called down-moving condition), when the angle measured by the goniometer reached one of the preset angle values $(\alpha 1, \alpha 2, \alpha 3)$. At this time, an electrical signal was automatically generated by the goniometer and triggered the onset of the electrical stimulation. Ninety-six trials (2 blocks, 3 angles, 2 movement directions, and 8 repetitions) were acquired. A minimum time of $30 \mathrm{~s}$ was kept as intertrial interval. During this interval, the subjects were asked to keep the arm relaxed. The voluntary movement condition was preceded and followed by a HBR recording session in the static condition ( 4 repetitions $\times 3$ stimulation positions before and after voluntary movement, for a total of 24 trials). This latter 

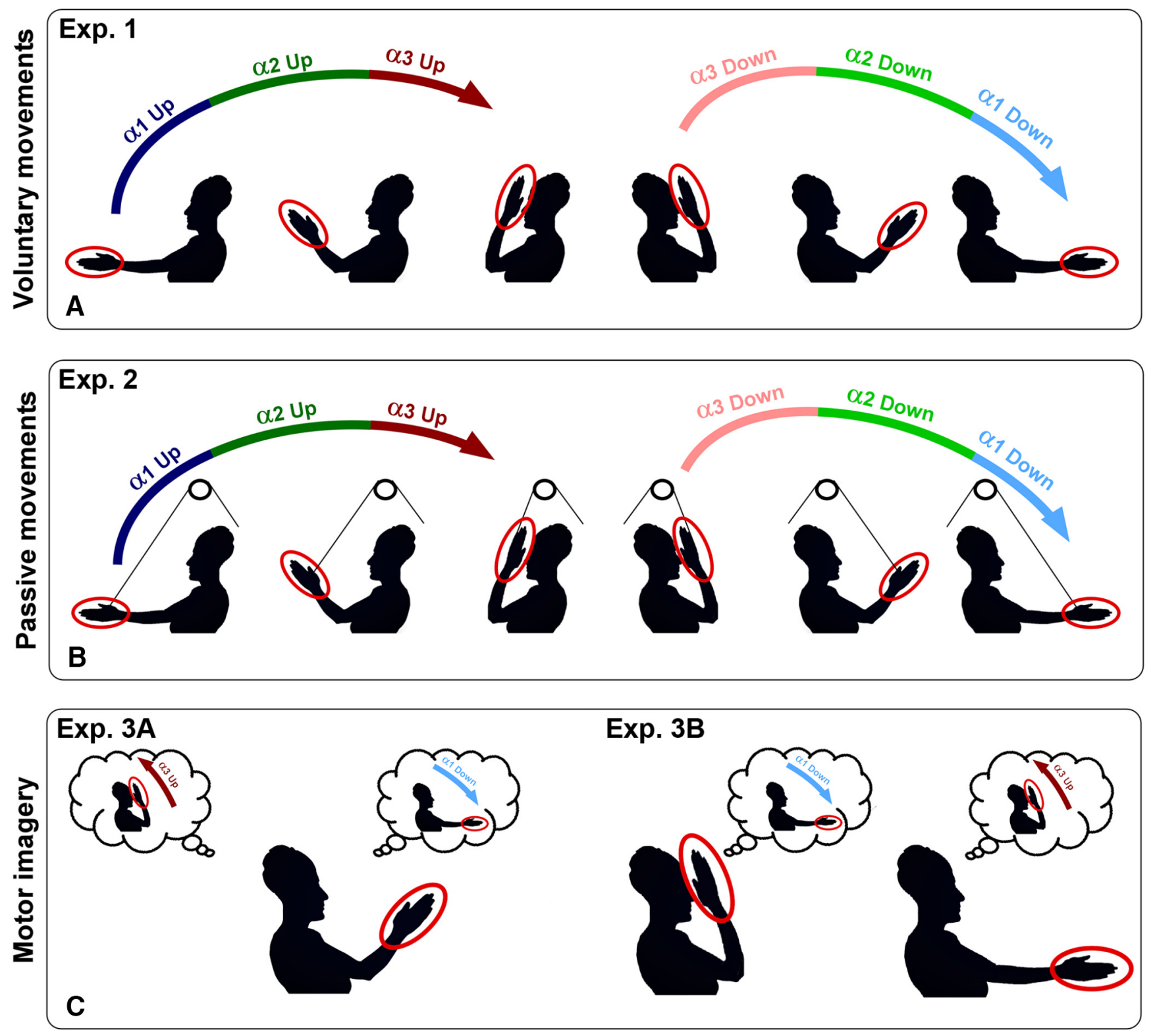

Figure 1. Experimental setup. $\boldsymbol{A}$, Experiment 1, during which participants voluntarily performed either a flexion- extension or an extension-flexion movement of the elbow: while moving up toward (up) or down far from (down) the face, they received an electrical stimulation in three preset positions: far $(\alpha 1)$, intermediate $(\alpha 2)$, and near ( $\alpha 3$ ). $\boldsymbol{B}$, Experiment 2 shows the pulley system used by the experimenter to induce the passive flexion- extension (up) and extension-flexion (down) movements to participants' right forearm. The electrical stimulation was delivered in the same conditions as in Experiment 1. C, Experiments 3A and 3B. In Experiment 3A, the subject kept a static position corresponding to $\alpha 2$ and imagined either an elbow flexion movement toward the face (up, from $\alpha 2$ to $\alpha 3$ ) or an elbow extension movement (down, from $\alpha 2$ to $\alpha 1$ ). In Experiment 3B, the subject imagined either a flexion or an extension movement starting from a static hand position corresponding to $\alpha 1$ (up, from $\alpha 1$ to $\alpha 3$ ) and $\alpha 3$ (down, from $\alpha 3$ to $\alpha 1$ ), respectively.

condition was introduced here and in each of the following experiments to test whether subjects' HBR responses in the three stimulation positions were comparable in the different days. Furthermore, this evaluation allowed testing possible effects on HBR amplitude due to habituation.

Experiment 2. Passive movement (Fig. 1B). This experiment was performed to test the role that afferent (i.e., visual and proprioceptive signals) inputs could play in modulating the HBR response. Participants were asked to keep the right arm completely relaxed in a plastic splint while the experimenter moved it by means of a transparent wire connected to the splint and a pulley system. The passive movement was an elbow flexion-extension of the right arm. A second transparent wire was attached to the opposite site of the hand support (hand back) and adjusted in length to avoid that the distance between the hand and the face of the participant was $<4 \mathrm{~cm}$. At the beginning, the experimenter asked the participant to perform an elbow flexion and extension movement at natural velocity. When the experimenter thought to have understood participant's natural movement velocity, she moved participants' forearm and verbally questioned the subject if he/she felt the movement velocity similar to his/her velocity. After participant agreement, the experiment started. No differences were found between angular movement velocities evaluated in Experiments 1 and 2 (mean \pm SD: Experiment 1, $100.13 \pm 37 \mathrm{deg} / \mathrm{s}$; and Experiment 2, $99.58 \pm 23 \mathrm{deg} / \mathrm{s} ; p=0.95)$.

The electrical stimulation was delivered during the passive movement when the angle measured by the goniometer reached the preset angle values $(\alpha 1, \alpha 2, \alpha 3)$ during either elbow flexion (up-moving condition) or extension (down-moving condition) movements. As in Experiment 1, an electrical signal, automatically generated by the goniometer, triggered the onset of the electrical stimulation. Different from Experiment 1, to reduce participants' expectancy, we introduced catch trials. We did not introduce the blocks paradigm used in Experiment 1 because in that condition no significant difference was found between the two blocks (see Results). This allowed us to dramatically reduce the number of trials. 
Excluding the catch trials, a total of 48 trials ( 3 angles, 2 directions, 8 repetitions) were acquired. The passive movement condition was preceded and followed by a HBR recording session in the static condition (4 repetitions $\times 3$ stimulation positions before and after passive movement, for a total of 24 trials).

Experiment 3A. Motor imagery from intermediate position (Fig. 1C). Before starting the experimental procedure, all the participants completed the Italian version of the Movement Imagery Questionnaire (Hall and Martin, 1997) to assess their ability to form kinesthetic and visual images. The Movement Imagery Questionnaire is an 8-item self-report questionnaire, in which participants rated the vividness of their mental representations using two 7-point scales (associated with visual and kinesthetic imagery): 1, "really easy to feel/see" to 7, "really difficult to feel/ see." All participants considered it fairly easy to form motor images, and the scores indicated that they possessed good motor imagery abilities (mean $\pm \mathrm{SD}=18.8 \pm 5.55)$. After that, they were instructed to put the arm in $\alpha 1$ and $\alpha 3$ positions to memorize them. Then, they were asked to keep the right arm in the position corresponding to $\alpha 2$ (intermediate position) and to kinesthetically imagine the right arm making a flexion (up-moving condition, from $\alpha 2$ to $\alpha 3$ ) or an extension (down-moving condition, from $\alpha 2$ to $\alpha 1$ ) movement at spontaneous velocity. At the beginning, for few trials, during motor imagery, participants had to verbally report when the upper arm reached $\alpha 1$ or $\alpha 3$. When the experimenter learned the time used by the participant to imagine to move toward one of the two positions, the experiment started. After each trial, the subject was questioned on whether the electrical stimulation was administered in correspondence to the position set for the current trial ( $\alpha 1$ or $\alpha 3$ ), and imagined by the subject. In case of mismatch, the trial was repeated. As in Experiment 2, catch trials were introduced to reduce participants' expectancy of the stimulus. Excluding the catch trials, a total of 16 trials were recorded for each subject (2 imagined directions, 8 repetitions). The motor imagery condition was preceded and followed by a HBR recording session in the static condition ( 4 repetitions $\times 3$ stimulation positions before and after motor imagery, for a total of 24 trials).

Experiment 3B. Motor imagery from near and far positions (Fig. 1C). This experiment was performed to assess whether and how motor imagery influences the HBR response when the hand was close to or far from the face. A group of participants, different from those who performed previous experiments, were instructed to put the arm in $\alpha 1, \alpha 2$, or $\alpha 3$, and to kinesthetically imagine one of the following movements at spontaneous velocity: a flexion movement from $\alpha 2$ to $\alpha 3$ (MI $\alpha 2$ to $\alpha 3$ ), an extension movement from $\alpha 2$ to $\alpha 1$ (MI $\alpha 2$ to $\alpha 1$ ) (the previous conditions replicated those proposed in Experiment 3A), a flexion movement from $\alpha 1$ to $\alpha 3$ (MI $\alpha 1$ to $\alpha 3$ ), and an extension movement from $\alpha 3$ to $\alpha 1$ (MI $\alpha 3$ to $\alpha 1$ ). As in Experiment 3A, at the beginning, participants had to verbally report when the upper arm reached the imagined final position to teach the experimenter about the time used to imagine the movement. After each trial, in case of mismatch reported by the participant between the arrival of the electrical stimulation and the stimulation position set for the current trial, the test was repeated. The four experimental conditions were executed in random order together and interleaved with catch trials. Excluding the catch trials, a total of 32 trials were recorded for each subject ( 4 imagined movements $\times 8$ repetitions). The motor imagery condition was preceded and followed by a HBR recording session in the static condition ( 4 repetitions $\times 3$ stimulation positions before and after motor imagery, for a total of 24 trials).

\section{Data processing and statistical analysis}

A custom-made MATLAB software (The MathWorks) was used to process the EMG signals. EMG signals from each participant were filtered and rectified. HBR responses were averaged separately in each condition and for each participant. Trials with an abnormal EMG activity preceding the HBR response were discarded by the analysis. The area under the curve (AUC, $\mathrm{mV} \times \mathrm{ms}$ ) of each HBR average waveform was considered as outcome parameter. To compute AUC in each average EMG trace, the software automatically analyzed a $130 \mathrm{~ms}$ time interval from the stimulus onset that always contained the subject's blink. The resulting curve was then integrated to compute AUC. In all experiments, data were averaged across ipsilateral and contralateral recording sides (right and left eyes) according to the previous analyses proposed in the literature (Sambo et al., 2012a).

In the preliminary experiment, AUC values acquired in the static condition were compared by mean of a repeated-measures ANOVA with position ( 3 levels: $\alpha 1, \alpha 2$, and $\alpha 3$ ) as within-subject factor. This analysis was used to confirm the literature and thus assess the reliability of our experimental setup. Further, it allowed us to identify the participants who showed a reproducible HBR response.

To compare the HBR responses in the preliminary experiment with those associated with the static condition in Experiments 1, 2, and 3A, the AUC values in the pre and post conditions of Experiments 1,2, and 3A were averaged. Then, these data were statistically compared by means of a repeated-measures ANOVA with position (3 levels: $\alpha 1, \alpha 2$, and $\alpha 3$ ) and session (4 levels: preliminary experiment, Experiment 1, Experiment 2, and Experiment $3 \mathrm{~A}$ ) as within-subject factors.

In Experiments 1, 2, and 3A, the AUC values measured in the static condition, before and after the different "dynamic" conditions, were subjected to three repeated-measures ANOVA (repeated-measures ANOVA) with position ( 3 levels: $\alpha 1, \alpha 2$, and $\alpha 3$ ) and time (2 levels: pre, post), as within-subject factors.

In Experiment 1, AUC data were analyzed by mean of repeatedmeasures ANOVA, with position ( 3 levels: $\alpha 1, \alpha 2$, and $\alpha 3$ ), block ( 2 levels: A and B), and movement direction (2 levels: up-moving, downmoving), as within-subject factors. Furthermore, the HBR responses during voluntary movements (AUC values averaged over the blocks) were compared with those acquired in the corresponding static condition (data were obtained by averaging AUC values evaluated in the pre and post conditions) by means of a repeated-measures ANOVA with condition (3 levels: static, up-moving, down-moving) and position (3 levels: $\alpha 1, \alpha 2$, and $\alpha 3$ ), as within-subject factors. In Experiment 2, AUC values were statistically analyzed by mean of a repeated-measures ANOVA with position ( 3 levels: $\alpha 1, \alpha 2$, and $\alpha 3$ ) and movement direction ( 2 levels: up-moving, down-moving). To compare AUC values evaluated during passive movement with those in the static condition (data were obtained by averaging AUC values evaluated in the pre and post conditions), a repeated-measures ANOVA with condition (3 levels: static, upmoving, down-moving) and position ( 3 levels: $\alpha 1, \alpha 2$, and $\alpha 3$ ), as within-subject factors, was performed. In Experiment $3 \mathrm{~A}$, a paired $t$ test (2 levels: up-moving, down-moving) was adopted to evaluate HBR responses during the imagination of flexion and extension movements. Further, we performed an additional analysis where a baseline condition, during which the HBR response in the static condition corresponding to $\alpha 2$ was directly compared with HBR amplitudes during MI in both upmoving and down-moving conditions (repeated-measures ANOVA, within factor condition, 3 levels: static $\alpha 2$, MI up-moving, MI downmoving). This allowed us to go deeper inside the mechanisms regulating the reflex response when the arm is actually in a static condition, but the motor system is involved in movement planning. In Experiment 3B, a repeated-measure ANOVA compared the AUC values in the static condition when the arm was positioned in $\alpha 1$ and $\alpha 3$ with those obtained during MI when the subject imagined to reach $\alpha 3$ starting from $\alpha 1$ ( $\alpha 1$ to $\alpha 3$ ), and imagined to reach $\alpha 1$ starting $\alpha 3$ ( $\alpha 3$ to $\alpha 1$ ) (within-subject factor condition, 2 levels: static and MI; within-subjects factor position, 2 levels: $\alpha 1$ and $\alpha 3$ ). Furthermore, we replicated the statistical analyses proposed in Experiment $3 \mathrm{~A}$ concerning the comparison among between MI $\alpha 2$ to $\alpha 3$ and MI $\alpha 2$ to $\alpha 1$ by means of a paired $t$ test and the comparison among static $\alpha 2$, MI $\alpha 2$ to $\alpha 3$, and MI $\alpha 2$ to $\alpha 1$ by means of a one-way ANOVA. Newmann-Keuls post hoc analysis was used to interpret significant interactions. Data in the text are reported as mean $\pm \mathrm{SE}$.

\section{Results \\ Preliminary experiment}

Static condition (Fig. 2). The statistical analysis showed a significant effect of the factor position $\left(F_{(2,18)}=7.49, p=0.004\right)$. Post hoc tests revealed a significant increase of AUC values in $\alpha 3(22.33 \pm 2.55$ $\mathrm{mV} \times \mathrm{ms})$ with respect to $\alpha 1(17.86 \pm 2.32 \mathrm{mV} \times \mathrm{ms}, p=0.02)$ and $\alpha 2(15.06 \pm 1.26 \mathrm{mV} \times \mathrm{ms}, p=0.003)$. These results confirmed the literature showing that when the stimulated arm is close to the face, 

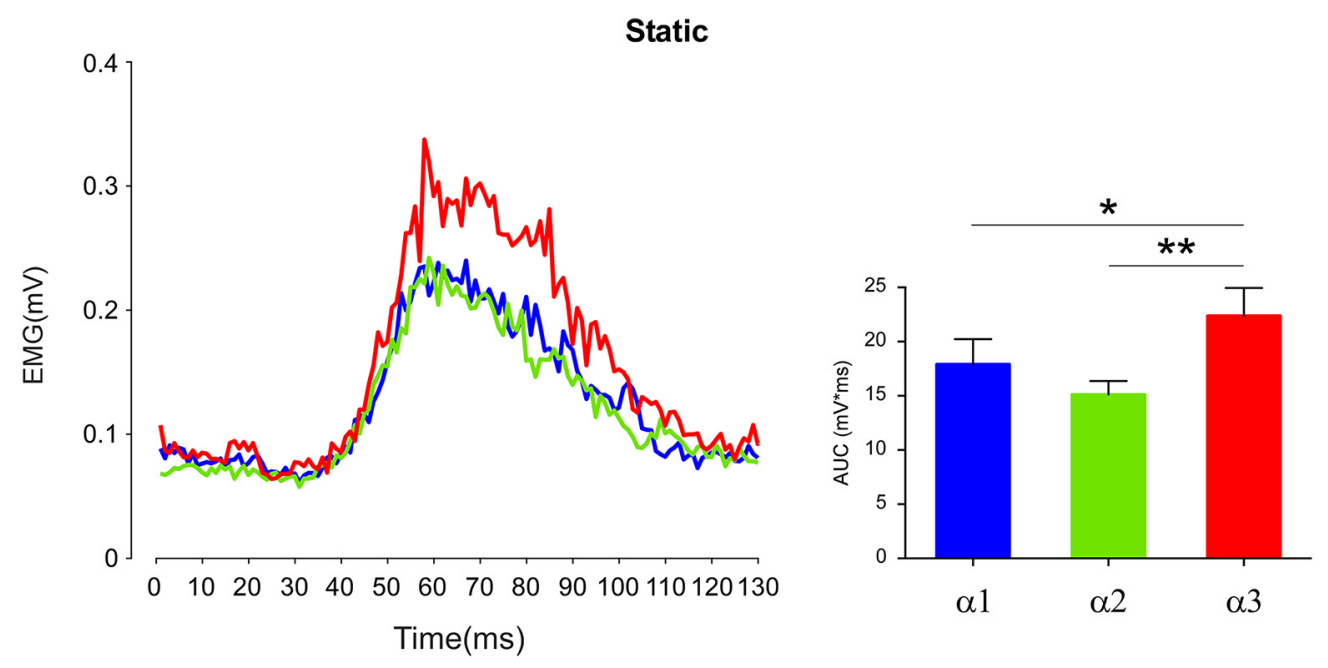

Figure 2. Preliminary experiment: static condition. Group-average, rectified HBR waveforms (left) and group-average HBR amplitudes (right, $A U C$, $m V \times m s)$ recorded when the arm was placed in the three stimulation positions: far $(\alpha 1)$, intermediate $(\alpha 2)$, and near $(\alpha 3)$. Error bars indicate standard error. ${ }^{* *} p<0.01,{ }^{*} p<0.05$.

inside the DPPS, the HBR magnitude is significantly higher than those evoked when the arm is in farther positions.

\section{Comparison among the static conditions}

The results of the repeated-measures ANOVA on AUC values related to the static conditions acquired in each testing session showed a significant effect of position $\left(F_{(2,18)}=17.87, p=\right.$ $0.00005)$, due to the significant increase of $\alpha 3$ with respect to the other stimulation positions ( $p$ always $<0.004)$. No differences appeared among the sessions $(p=0.33)$.

Repeated-measures ANOVA on AUC values evaluated in the static condition during Experiments 1,2, and 3A revealed an effect of the factor position (Experiment 1: $F_{(2,18)}=11.21, p=0.0006$; Experiment 2: $F_{(2,18)}=8.19, p=0.002$; Experiment $3 \mathrm{~A}: F_{(2,18)}=5.31$, $p=0.01$ ) due to the significant increase of the AUC values in $\alpha 3$ with respect to $\alpha 2$ and $\alpha 1$ ( $p$ always $<0.05$ ). No differences appeared in the HBR responses acquired in static condition before and after the "dynamic" experimental conditions ( $p$ always $>0.2$ ).

\section{Experiment 1}

Voluntary movement. Single-subject average data in each conditions are shown in Figure 3. Repeated-measures ANOVA showed that movement direction $\left(F_{(1,9)}=5.66, p=0.04\right)$ as well as position $\left(F_{(2,18)}=6.94, p=0.006\right)$ significantly affected the amplitude of the HBR responses, and a significant interaction between these two factors was found $\left(F_{(2,18)}=8.34, p=0.003\right)$. Post hoc analysis showed that, during an elbow flexion movement (up-moving condition), there was a significant increase of the HBR value in $\alpha 3(12.3 \pm 1.7 \mathrm{mV} \times \mathrm{ms})$ with respect to $\alpha 2(8 \pm$ $1.1 \mathrm{mV} \times \mathrm{ms}, p=0.0003)$ and $\alpha 1(8.7 \pm 1 \mathrm{mV} \times \mathrm{ms}, p=0.001)$ (Fig. 4A). Differently, during elbow extension movements (down-moving condition), no difference in the AUC values was found in the three stimulation positions $(\alpha 1=9.3 \pm 1.3 \mathrm{mV} \times$ $\mathrm{ms}, \alpha 2=8.9 \pm 1.1 \mathrm{mV} \times \mathrm{ms}, \alpha 3=9.3 \pm 1.1 \mathrm{mV} \times \mathrm{ms}, p$ always $>0.7$ ) (Fig. $4 B$ ). Further, the HBR response in $\alpha 3$ when moving up to the face was significantly higher than that observed at the same position when the hand moved far from the face $(p=$ $0.001)$. Finally, no difference between the two experimental blocks (i.e., elbow flexion-extension and elbow extension-flexion) was found ( $p=0.23)$.

Further, when we compared HBR responses in the static condition and during voluntary movements, significant main effects of condition $\left(F_{(2,18)}=32.94, p=0.000001\right)$ and position $\left(F_{(2,18)}=\right.$ $13.64, p=0.0002)$ were found. Post hoc analysis revealed that AUC values in the static condition were significantly higher than those obtained during voluntary up and down movements ( $p$ always $<0.0005)$. Concerning the factor position, the HBR response in $\alpha 3$ was significantly higher than in $\alpha 1$ and $\alpha 2$ ( $p$ always $<0.001$ ). Furthermore, we found a significant interaction between condition and position $\left(F_{(4,36)}=4.52, p=0.005\right)$, suggesting that the position effect (i.e., a greater response in $\alpha 3$ position with respect to both $\alpha 1$ and $\alpha 2$ ) was present only in the static condition ( $p$ always $<0.0002$ ) and in up movements (respectively, $p=0.02, p=0.007$ ), whereas no position effect pertained to the down movements.

\section{Experiment 2}

Passive movement. The statistical analysis showed a significant interaction between position and movement direction $\left(F_{(2,18)}=\right.$ $6.91, p=0.006)$. As in the case of voluntary movement, during passive elbow flexion movements (up-moving condition) the magnitude of the HBR responses significantly increase when the hand was near the face $(\alpha 3=11.8 \pm 1.8 \mathrm{mV} \times \mathrm{ms})$ with respect to $\operatorname{far}(\alpha 1=6.5 \pm 0.9 \mathrm{mV} \times \mathrm{ms}, p=0.007)$ and intermediate $(\alpha 2=7.3 \pm 0.6 \mathrm{mV} \times \mathrm{ms}, p=0.02)$ positions (Fig. $5 A$ ), whereas during extension movements (down-moving condition) no difference was found among the three hand positions $(\alpha 1=9.6 \pm$ $0.9 \mathrm{mV} \times \mathrm{ms}, \alpha 2=8.1 \pm 0.8 \mathrm{mV} \times \mathrm{ms}, \alpha 3=8.2 \pm 0.9 \mathrm{mV} \times$ ms, $p$ always $>0.3$ ) (Fig. $5 B$ ). Finally, the HBR response in $\alpha 3$ when the arm was passively moved up-to the face was significantly higher than that observed at the same position when the arm was moved far from the face $(p=0.03)$.

When we compared HBR responses in the static condition and during passive movement, significant main effects of condition $\left(F_{(2,18)}=14.28, p=0.0002\right)$ and position $\left(F_{(2,18)}=7.6, p=0.004\right)$ were found. Post hoc analysis revealed that AUC values in the static condition were significantly higher than those obtained during passive movements ( $p$ always $<0.0006$ ), and that the HBR response in $\alpha 3$ was significantly higher than in $\alpha 1$ and $\alpha 2$ ( $p$ always $<0.0041$ ). Furthermore, we found a significant interaction between condition and position $\left(F_{(4,36)}=4.14, p=0.007\right)$, suggesting that the position effect (i.e., a greater response in $\alpha 3$ position with respect to both $\alpha 1$ and $\alpha 2$ ) was present only in static condition ( $p$ always $<0.03)$ and in up movements (respectively, $p=0.002, p=0.006$ ), whereas no position effect pertained to the down movements. 


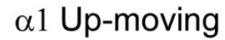

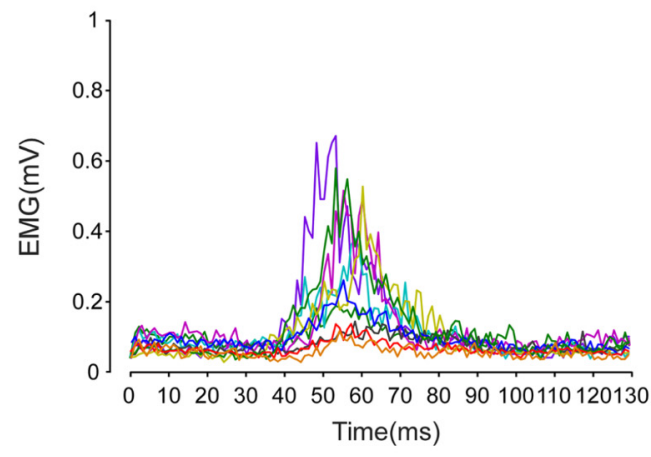

a2 Up-moving

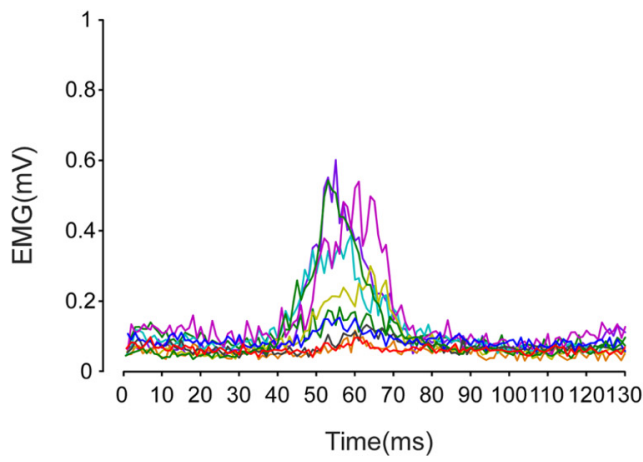

a3 Up-moving

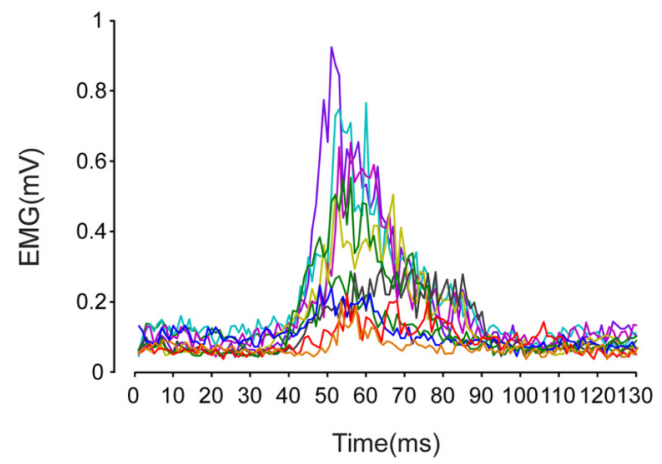

a1 Down-moving

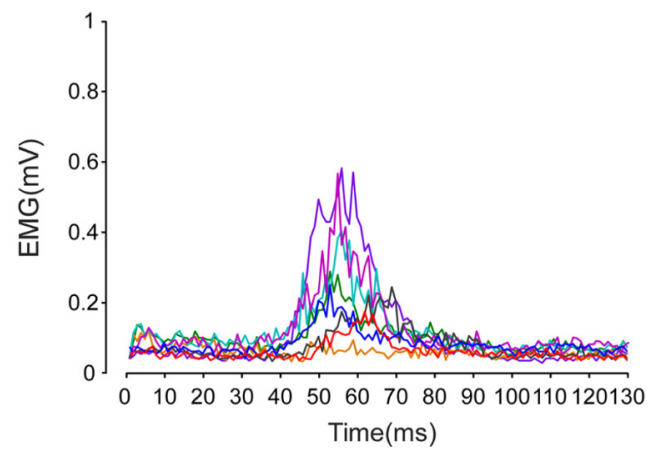

a2 Down-moving

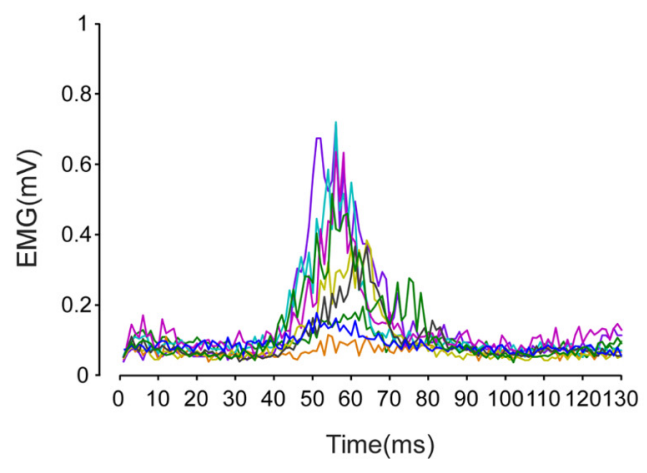

a3 Down-moving

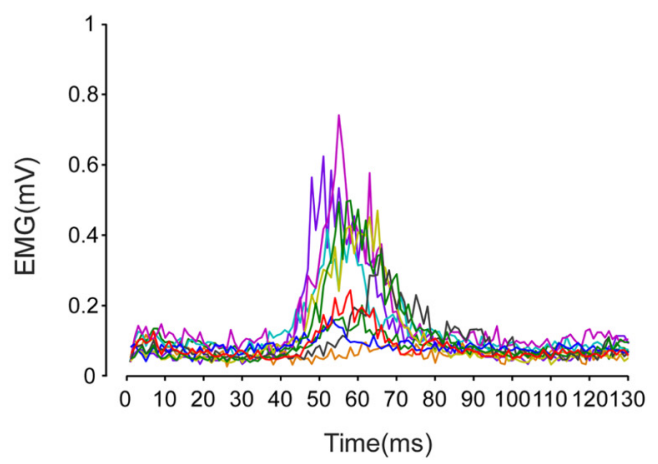

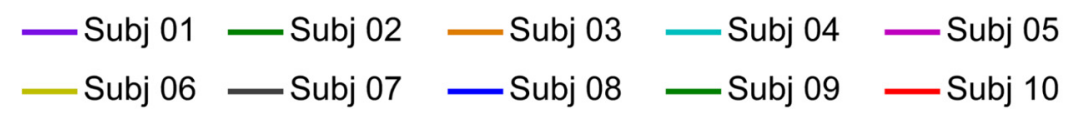

Figure 3. Experiment 1: voluntary movements. Rectified and superimposed average EMG traces (mean over the blocks and recording sites) of each participant for the near ( $\alpha 1)$, intermediate $(\alpha 2)$, and far ( $\alpha 3)$ positions when participants performed up movement toward the face (up-moving) or down movement far from the face (down-moving).

\section{Experiment 3A}

Motor imagery from intermediate position (Fig. 6). The results of the paired $t$ test showed a significant effect of the direction of the imagined movement: when participants imagined a flexion movement (up-moving condition), from the intermediate to the near position, the HBR responses were significantly higher $(10.9 \pm 0.9 \mathrm{mV} \times \mathrm{ms})$ than when they imagined to extend their arm toward the far position $(9.9 \pm 0.7 \mathrm{mV} \times \mathrm{ms})(t=3.04, p=$ $0.01)$. Further, when these conditions were directly compared with a situation in which the subject kept the arm fixed in $\alpha 2$, the ANOVA showed a significant effect of the factor condition $\left(F_{(2,18)}=15.09, p=0.0001\right)$. The post hoc analysis revealed that MI conditions, regardless of the imagined movement direction, induced a reduction of the HBR response ( $p$ always $<0.0007$ ).

\section{Experiment 3B}

Motor imagery from near and far positions. The results of the paired $t$ test between the motor imagery $\alpha 2$ to $\alpha 3$ and $\alpha 2$ to $\alpha 1$ showed that AUC values associated with the imagined flexion movement ( $\alpha 2$ to $\alpha 3,11.3 \pm 1.0 \mathrm{mV} \times \mathrm{ms}$ ) were significantly higher $(t=3.07, p=0.01)$ than those associated with the extension movement ( $\alpha 2$ to $\alpha 1,10.2 \pm 0.7 \mathrm{mV} \times \mathrm{ms}$ ), confirming the results described in Experiment 3A. Further, we confirmed also that, when these conditions were directly compared with AUC values in the static condition corresponding to $\alpha 2(14.1 \pm 0.9$ $\mathrm{mV} \times \mathrm{ms})$, a significant effect of condition appeared $\left(F_{(2,22)}=\right.$ $11.38, p=0.0004)$. The post hoc analysis revealed that MI conditions, regardless of the imagined movement direction, induced a reduction of the HBR response ( $p$ always $<0.01$ ). 


\section{Voluntary movements}
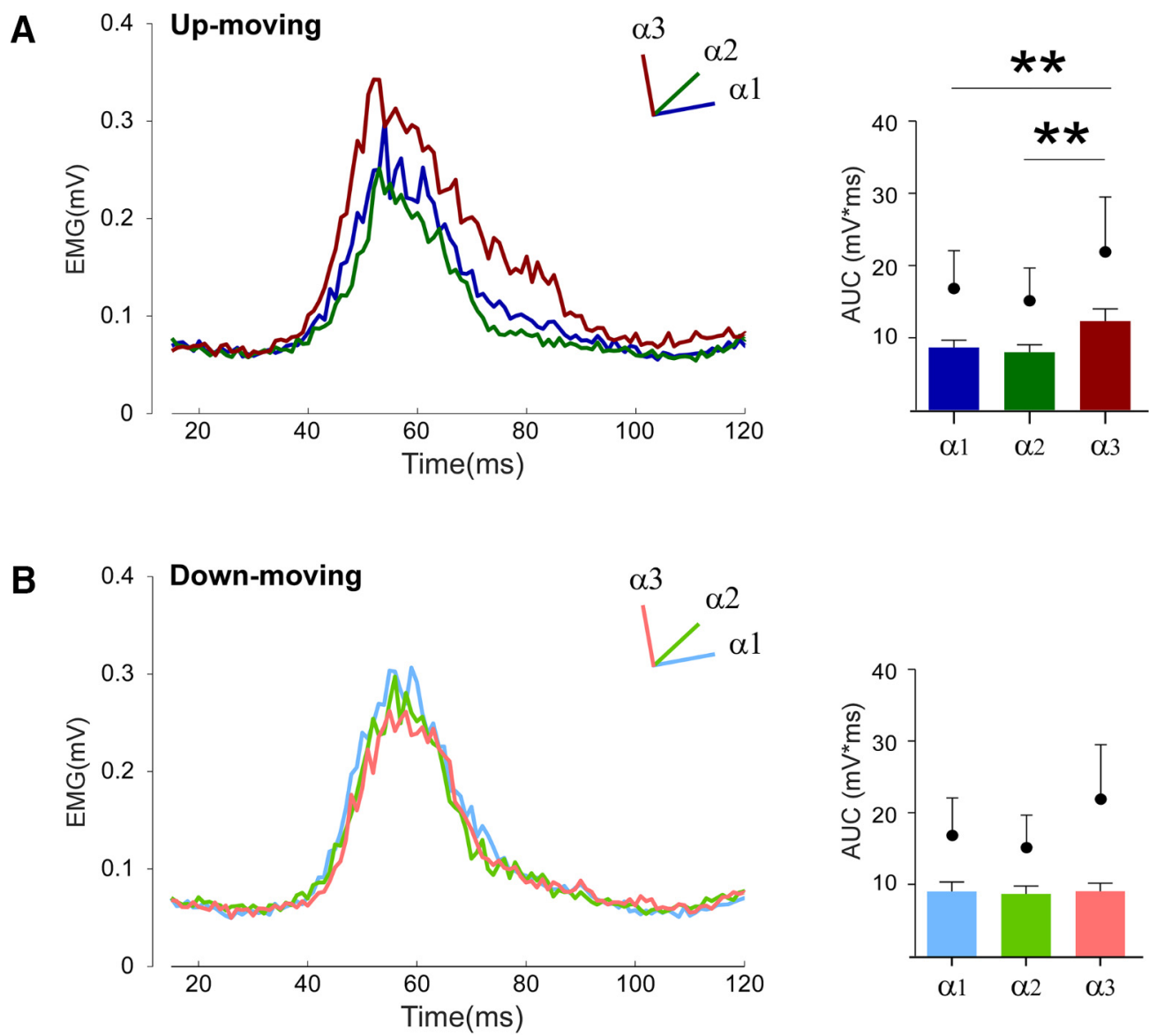

Figure 4. Experiment 1: voluntary movements. Group-average, rectified HBR waveforms (left) and the group-average HBR amplitudes (right, AUC, $m V \times m s)$ in the up-moving $(A)$ and down-moving $(\boldsymbol{B})$ conditions in the three stimulation positions: far $(\alpha 1)$, intermediate $(\alpha 2)$, and near $(\alpha 3)$. Black dots indicate group-average AUC values in the corresponding static condition. Error bars indicate standard error. ${ }^{* *} p<0.01$.

When the AUC values in MI conditions from $\alpha 1$ to $\alpha 3$ and from $\alpha 3$ to $\alpha 1$ were compared with those corresponding to the static condition in $\alpha 1$ and $\alpha 3$ (Fig. 7), ANOVA showed a significant main effect of condition $\left(F_{(1,11)}=19.86, p=\right.$ 0.001 ), where AUC mean values in the static condition were higher than that recorded during MI. Further, a significant interaction condition $\times$ position $\left(F_{(1,11)}=9.99, p=0.001\right)$ was found. The post hoc examinations showed that, in the static condition, the HBR response was significantly higher in $\alpha 3(17.7 \pm 1.5 \mathrm{mV} \times \mathrm{ms})$ than in $\alpha 1(13.7 \pm 1.1 \mathrm{mV} \times \mathrm{ms})$ $(p=0.0003)$, whereas no differences appeared between MI $\alpha 1$ to $\alpha 3(10.9 \pm 0.8 \mathrm{mV} \times \mathrm{ms})$ and $\alpha 3$ to $\alpha 1(11.7 \pm 1.1 \mathrm{mV} \times$ ms) $(p=0.31)$. Furthermore, AUC values in the static conditions were significantly higher than those obtained during MI starting from the same hand positions (static $\alpha 1$ vs MI $\alpha 1$ to $\alpha 3, p=0.008$; static $\alpha 3$ vs MI $\alpha 3$ to $\alpha 1, p=0.0002$ ).

\section{Discussion}

In this study, we sought for evidence that the predictive motor system can modulate a defensive response, the HBR. The preliminary experiment performed in the static condition showed that the HBR is significantly enhanced when one's own stimulated hand is located inside the DPPS of the face, and this result is in agreement with the previously described "hand position" effect (Sambo et al., 2012a, b; Sambo and Iannetti, 2013).
Experiment 1 extended beyond the previous research on DPPS, passing from static to dynamic conditions. First, we found a significant HBR enhancement in the near position also in dynamic conditions (i.e., when the moving stimulated hand entered the DPPS of the face). It is worth noting that, as previously suggested for the static condition (Sambo and Iannetti, 2013), the HBR response was not linearly enhanced through the three stimulation positions, but a safety boundary exists: only inside this boundary the stimulus is potentially dangerous. Indeed, in both static and dynamic conditions, comparable HBR responses were found when stimuli were received in far $(\alpha 1)$ and intermediate $(\alpha 2)$ positions. But, mostly relevant is the HBR enhancement in the near $(\alpha 3)$ position with respect to $\alpha 1$ and $\alpha 2$ only when the hand was moving toward and not down from the face, demonstrating the existence of a "hand movement direction" effect in HBR modulation, and suggesting that the space representation is dynamically shaped by the movement. Thus, what is crucial in HBR modulation in dynamic conditions it is not the actual position of the stimulated hand, but the final position where the hand is expected to be at the end of the movement. The lack of an increase of HBR response when the hand from the far position moves toward the face might suggest that the dynamic shaping of the DPPS interacts with other aspects defining the safety boundary around the body. As mentioned above, the HBR enhance- 


\section{Passive movements}
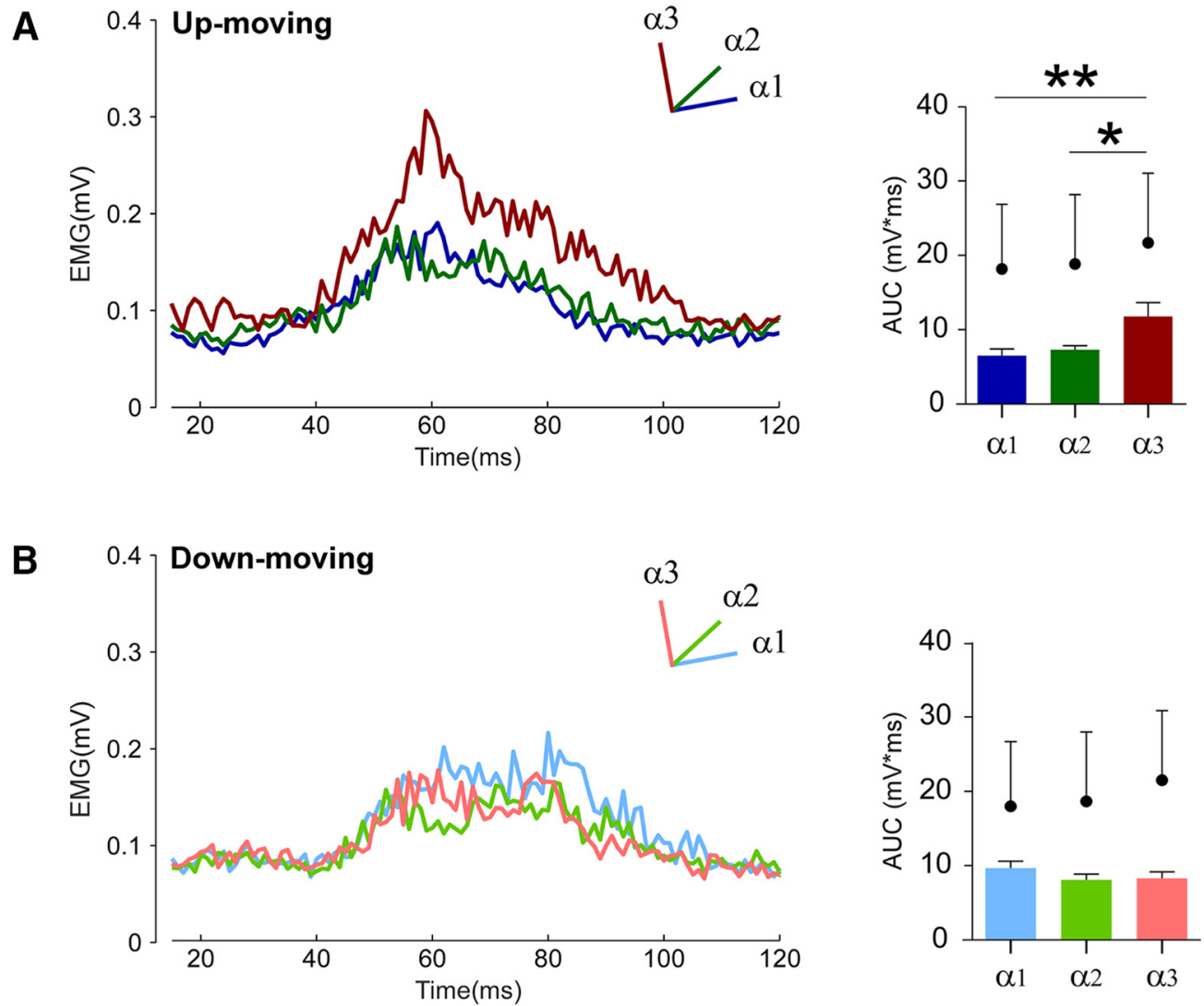

Figure 5. Experiment 2: passive movements. Group-average, rectified HBR waveforms (left) and the group-average HBR amplitudes (right, AUC, $\mathrm{mV} \times \mathrm{ms})$ in the up-moving $(\boldsymbol{A})$ and down-moving $(\boldsymbol{B})$ conditions in the three stimulation positions: far $(\alpha 1)$, intermediate $(\alpha 2)$, and near $(\alpha 3)$. Black dots indicate group-average AUC values in the corresponding static condition. Error bars indicate standard error. ${ }^{*} p<0.05 .{ }^{* *} p<0.01$.

\section{Motor imagery}
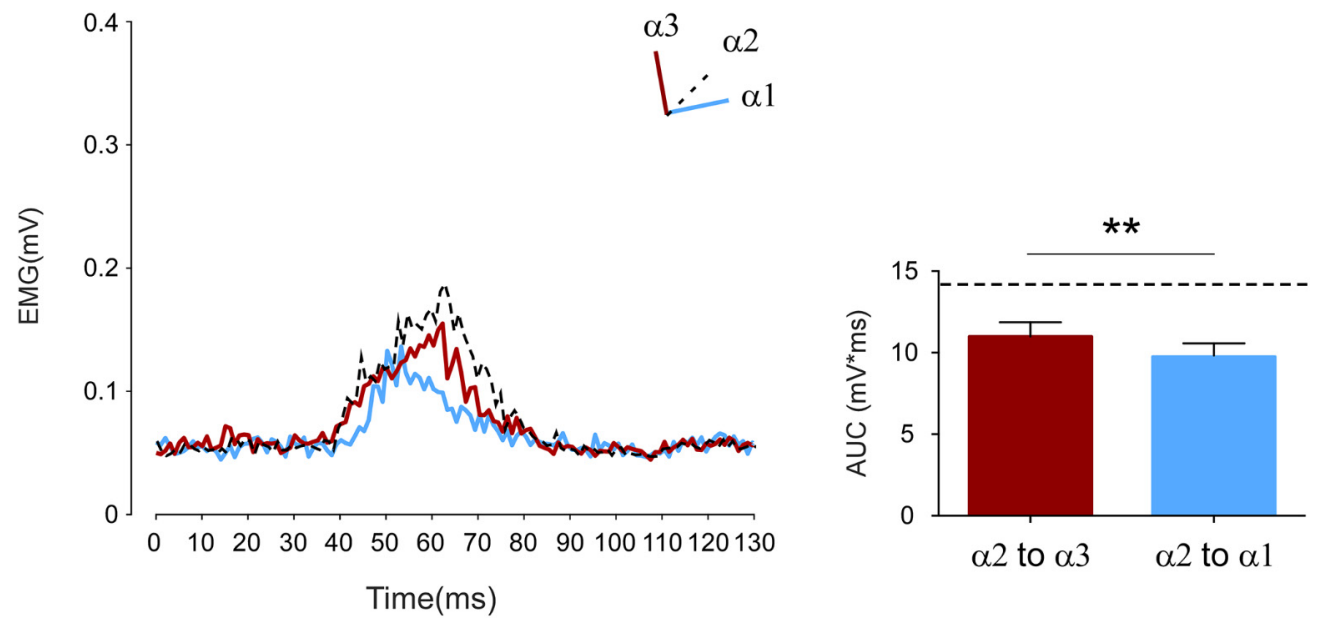

Figure 6. Experiment $3 \mathrm{~A}$ : motor imagery. Group-average, rectified HBR waveforms (left) and group-average HBR amplitudes (right, AUC, $\mathrm{mV} \times \mathrm{ms}$ ) when participants were asked to imagine to move the forearm from the intermediate to the near position ( $\alpha 2$ to $\alpha 3$ ) and from the intermediate to the far position $(\alpha 2$ to $\alpha 1)$. Dashed lines indicate the HBR waveform (left) and response amplitude (right) obtained in static condition corresponding to $\alpha 2$. Error bars indicate standard error. ${ }^{* *} p<0.01$.

ment does not have a linear trend (i.e., a significant difference was present only between near position and the other positions). Thus, coherently, the directional modulation could occur only in the near position. We might also speculate that the "hand move- ment direction" effect can operate as an "energy saving" mechanism that may reduce the defensive response when the stimulus is implicitly perceived as not risky, namely, when the predictive motor system informs that the stimulus has been administered to 

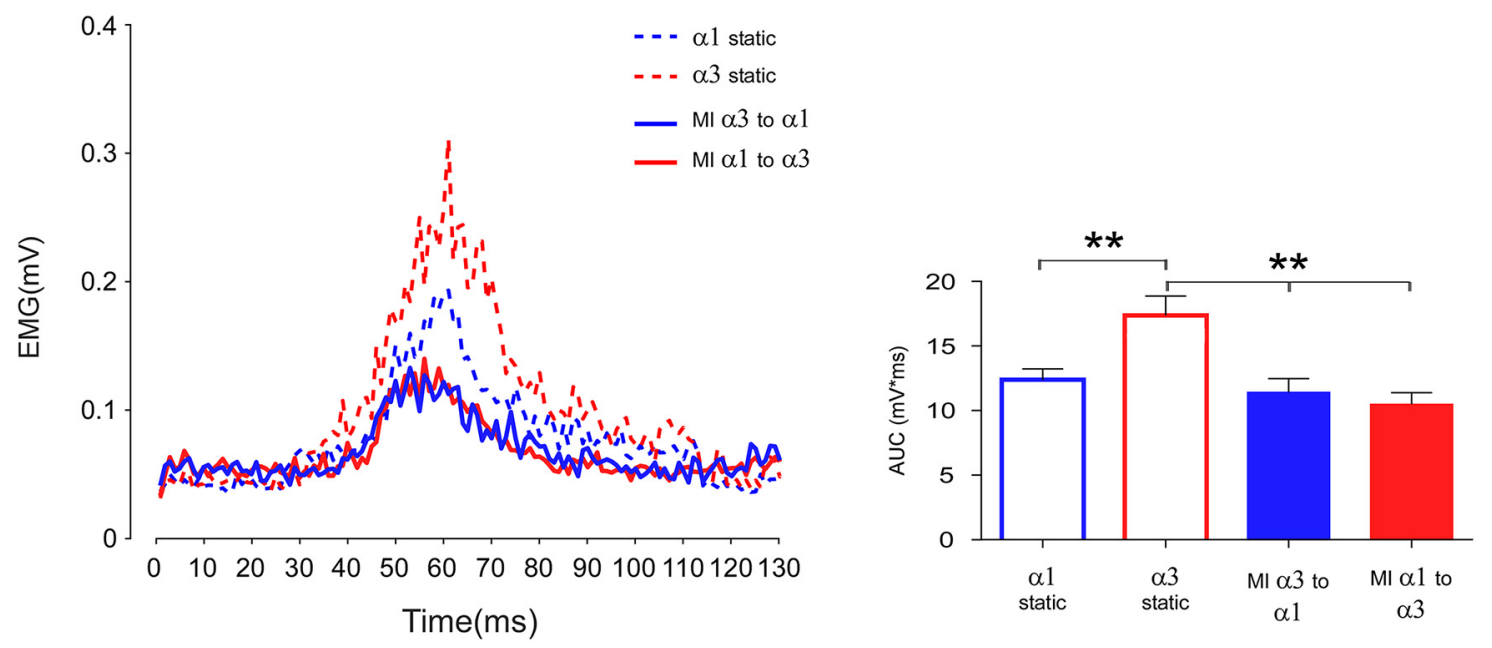

Figure 7. Experiment 3B: motor imagery. Group-average, rectified HBR waveforms (left) and group-average HBR amplitudes (right, AUC, $\mathrm{mV} \times \mathrm{ms}$ ) in the static condition corresponding to $\alpha 1$ (blue dotted line and blue empty column) and $\alpha 3$ (red dotted line and red empty column), and when participants were asked to imagine to move the forearm from the far to the near position ( $\alpha 1$ to $\alpha 3$, red straight line and red column) and from the near to the far position ( $\alpha 3$ to $\alpha 1$, blue straight line and blue column). Error bars indicate standard error. ${ }^{* *} p<0.01$.

the hand moving away from the face and, consequently, when "the near is becoming far."

During voluntary movements, two sources of information are available to the motor system to estimate the arm's final state: the sensory inflow, like the information coming from vision and proprioception, and the motor outflow (i.e., the copy of the motor commands). The results of Experiment 1 could not disambiguate between the role of these kinds of information in modulating the defensive response. One possibility is that this directional effect only pertains to the voluntary movements, when both the afferent and the efferent information is present. Alternatively, the directional effect could be present also when the afferent and the efferent sources of information are dissociated, as in the passive movement (Experiment 2) and in the motor imagery tasks (Experiment $3 \mathrm{~A}$ and $3 \mathrm{~B}$ ). Our data verified this second hypothesis, showing a directional effect on the HBR modulation during both passive movements and motor imagery tasks.

Similarly to the voluntary movement, in Experiment 2, dealing with passive movements, the HBR response increased when the hand received the stimulus near to the face only in the upmoving condition, whereas no difference among the three hand positions was found in the down-moving condition. This means that the modulation of the HBR response occurred also when only the afferent information (coming from vision and proprioception) was available to the system for predicting the consequence of the movement.

The results of the motor imagery tasks (Experiment $3 \mathrm{~A}$ and Experiment $3 \mathrm{~B}$ ) showed that the HBR response was significantly greater when the subject imagined to move up to than down from the face, although the arm was kept in the intermediate position $(\alpha 2)$ and the position remained unchanged during the experiment. When the hand was placed close to (near, $\alpha 3$ ) or far from (far, $\alpha 1)$ the face and subjects were asked to imagine to go down from ( $\alpha 3$ to $\alpha 1)$ or up to ( $\alpha 1$ to $\alpha 3)$ the face, respectively, no difference was observed between HBR amplitudes (Experiment 3B). Although these two hand positions are shown to evoke divergent responses in static condition, the motor imagery was able to abolish this difference, a result that underlines the role played by MI in modulating the reflex response. Moreover, when the hand positions corresponded to $\alpha 3$ and the subjects imagined to move far from the face ( $\alpha 3$ to $\alpha 1$ ), the HBR response significantly decreased with respect to the corresponding static position. In agreement with the results of voluntary and passive movement conditions, these findings might be explained as a downregulation of the HBR response when planning to move far from the face, albeit the hand was inside the DPPS. Therefore, results from the motor imagery tasks strongly support that the modulation of the defensive response occurred also when no actual movements were executed, but when only the efferent information (i.e., the efference copy of the motor program) was available to predict the final consequences of the movement. Converging evidence suggests that imagined and actual movements trigger similar motor representations (Jeannerod and Decety, 1995; Gentili et al., 2004; Gandrey et al., 2013) and share overlapping neural substrates (Grèzes and Decety, 2001; Jeannerod, 2001; Lotze and Halsband, 2006; Filimon et al., 2007; Hanakawa et al., 2008; Hetu et al., 2013; Bonzano et al., 2016). In particular, motor imagery would engage the same internal forward models (i.e., the neural mechanisms that mimic the causal flow of the physical process by predicting the future sensorimotor state that are involved in action execution) (Wolpert and Flanagan, 2001).

From an anatomical viewpoint, it has been proposed that the key mechanism generating the somatosensory-evoked blink reflex may exist before somatosensory signals enter the common blink interneuronal networks. One possibility is that the appearance of the HBR may depend on the level of activity of a gating mechanism that exerts inhibition on the inflow of the somatic input. Therefore, HBR modulation may be the result of the integration of facilitatory and inhibitory mechanisms within the brainstem exerted by higher centers, such as the basal ganglia, cerebellum, or cortex (Miwa et al., 1998). It has been proposed that the brainstem circuits mediating the HBR in humans undergo top-down modulation from higher order cortical areas (the polysensory zone in the precentral gyrus and the ventral intraparietal area) responsible for encoding the location of somatosensory stimuli in external space coordinates (Sambo et al., 2012b). Particularly relevant for the dynamic context of our study is that the application of an inhibitory repetitive transcranial magnetic stimulation on the hand motor area determined a longlasting reduction of excitability of the R2 component of the blink reflex (De Vito et al., 2009), probably mediated by direct projections of motor cortical areas to the lateral medullary reticular 
formation (Kuypers, 1958). Furthermore, in the MI context, the influence of cortical areas on brainstem neural circuits has been recently demonstrated during MI of a dynamic balance task (Ferraye et al., 2014). Thus, we can speculate that, when subjects move or imagine moving the hand throughout different positions inside the DPPS, the movement might differently activate the descending pathways originating from motor and associative cortical areas. These areas can influence the blink premotor activity, before entering the common blink-reflex pathway, shaping the amplitude of the HBR response.

Finally, the higher values of HBR response observed in the static condition with respect to the dynamic conditions of the three experimental sessions might suggest the use of two distinct neural mechanisms in modulating HBR in static and in dynamic conditions. Indeed, for each stimulation position, a significantly reduced HBR was found in dynamic with respect to static conditions. The decrease of HBR response during movement can be explained as consequence of the sensory attenuation, according to which the sensory effects generated by one's own actions are attenuated compared with the same effects generated externally (Blakemore et al., 1999; Tsakiris and Haggard, 2003; Bays et al., 2006; Weiss et al., 2011). It is important to note that, in our experimental context, during voluntary and passive conditions, the participant's movement triggered the electrical stimulator. Thus, the resulting stimulus can be interpreted as a self-generated sensory effect. We can speculate that, in the context of DPPS, a stimulus on which I can exert a direct control is interpreted as less dangerous and produces a lower defensive physiological response with respect to an externally generated stimuli, that is, by definition, outside from the subject's control. A similar explanation could account for the results of the motor imagery condition. Indeed, recent studies showed that the sensory attenuation occurs not only during action execution, but also during action preparation and planning (e.g., Lange, 2009). Alternatively, the movement-related HBR decrease can be explained by the different processing of the proprioceptive inputs in static and dynamic conditions. We can suggest that, in the static condition, the proprioceptive inputs can give an exact information about the position of the hand with respect to the face. Conversely, when a subject is moving, the rapidly changing inputs generated during hand movements can make it more difficult to estimate the hand position and therefore might reduce the amplitude of the reflex response. It is worth noting that the movement-related HBR decrease with respect to the static conditions is particularly relevant for the motor imagery condition, where, although the subjects did not actually move, the mere movement planning was sufficient to modify the reflex response.

Together, these findings provide physiological evidence for the role of the predictive motor system in dynamically shaping the DPPS during movement.

\section{References}

Bays PM, Flanagan JR, Wolpert DM (2006) Attenuation of self-generated tactile sensations is predictive, not postdictive. PLoS Biol 4:e28. CrossRef Medline

Blakemore SJ, Frith CD, Wolpert DM (1999) Spatio-temporal prediction modulates the perception of self-produced stimuli. J Cogn Neurosci 11: 551-559. CrossRef Medline

Blakemore SJ, Wolpert DM, Frith CD (2002) Abnormalities in the awareness of action. Trends Cogn Sci 6:237-242. CrossRef Medline

Bonzano L, Roccatagliata L, Ruggeri P, Papaxanthis C, Bove M (2016) Frontoparietal cortex and cerebellum contribution to the update of actual and mental motor performance during the day. Sci Rep 6:30126. CrossRef
Cooke DF, Graziano MS (2003) Defensive movements evoked by air puff in monkeys. J Neurophysiol 90:3317-3329. CrossRef Medline

de Vignemont F, Iannetti GD (2015) How many peripersonal spaces? Neuropsychologia 70:327-334. CrossRef Medline

De Vito A, Gastaldo E, Tugnoli V, Eleopra R, Casula A, Tola MR, Granieri E, Quatrale R (2009) Effect of slow rTMS of motor cortex on the excitability of the Blink Reflex: a study in healthy humans. Clin Neurophysiol 120:174-180. CrossRef Medline

Ferraye MU, Debû B, Heil L, Carpenter M, Bloem BR, Toni I (2014) Using motor imagery to study the neural substrates of dynamic balance. PLoS One 9:e91183. CrossRef Medline

Filimon F, Nelson JD, Hagler DJ, Sereno MI (2007) Human cortical representations for reaching: mirror neurons for execution, observation, and imagery. Neuroimage 37:1315-1328. CrossRef Medline

Fossataro C, Sambo CF, Garbarini F, Iannetti GD (2016) Interpersonal interactions and empathy modulate perception of threat and defensive responses. Sci Rep 6:19353. CrossRef Medline

Gandrey P, Paizis C, Karathanasis V, Gueugneau N, Papaxanthis C (2013) Dominant vs. nondominant arm advantage in mentally simulated actions in right handers. J Neurophysiol 110:2887-2894. CrossRef Medline

Gentili R, Cahouet V, Ballay Y, Papaxanthis C (2004) Inertial properties of the arm are accurately predicted during motor imagery. Behav Brain Res 155:231-239. CrossRef Medline

Graziano MS, Cooke DF (2006) Parieto-frontal interactions, personal space, and defensive behavior. Neuropsychologia 44:2621-2635. CrossRef Medline

Grèzes J, Decety J (2001) Functional anatomy of execution, mental simulation, observation, and verb generation of actions: a meta-analysis. Hum Brain Mapp 12:1-19. CrossRef Medline

Haggard P (2005) Conscious intention and motor cognition. Trends Cogn Sci 9:290-295. CrossRef Medline

Hall CR, Martin KA (1997) Measuring movement imagery abilities: a revision of the Movement Imagery Questionnaire. J Ment Imag 21:143-154.

Hanakawa T, Dimyan MA, Hallett M (2008) Motor planning, imagery, and execution in the distributed motor network: a time-course study with functional MRI. Cereb Cortex 18:2775-2788. CrossRef Medline

Hétu S, Grégoire M, Saimpont A, Coll MP, Eugène F, Michon PE, Jackson PL (2013) The neural network of motor imagery: An ALE meta-analysis. Neurosci Biobehav Rev 37:930-949. CrossRef Medline

Jeannerod M (2001) Neural simulation of action: a unifying mechanism for motor cognition. Neuroimage 109:103-109. CrossRef Medline

Jeannerod M, Decety J (1995) Mental motor imagery: a window into the representational stages of action. Curr Opin Neurobiol 5:727-732. CrossRef Medline

Kuypers HG (1958) Corticobulbar connexions to the pons and lower brainstem in man: an anatomical study. Brain 81:364-388. CrossRef Medline

Lange K (2009) Brain correlates of early auditory processing are attenuated by expectations for time and pitch. Brain Cogn 69:127-137. CrossRef Medline

Lotze M, Halsband U (2006) Motor imagery. J Physiol Paris 99:386-395. CrossRef Medline

Miwa H, Nohara C, Hotta M, Shimo Y, Amemiya K (1998) Somatosensoryevoked blink response: Investigation of the physiological mechanism. Brain 121:281-291. CrossRef Medline

Oldfield RC (1971) The assessment and analysis of handedness: the Edinburgh Inventory. Neuropsychologia 9:97-113. CrossRef Medline

Rizzolatti G, Fadiga L, Fogassi L, Gallese V (1997) The space around us. Science 277:190-191. CrossRef Medline

Sambo CF, Iannetti GD (2013) Better safe than sorry? The safety margin surrounding the body is increased by anxiety. J Neurosci 33:14225-14230. CrossRef Medline

Sambo CF, Forster B, Williams SC, Iannetti GD (2012a) To blink or not to blink: fine cognitive tuning of the defensive peripersonal space. J Neurosci 32:12921-12927. CrossRef Medline

Sambo CF, Liang M, Cruccu G, Iannetti GD (2012b) Defensive peripersonal space: the blink reflex evoked by hand stimulation is increased when the hand is near the face. J Neurophysiol 107:880-889. CrossRef Medline

Tsakiris M, Haggard P (2003) Awareness of somatic events associated with a voluntary action. Exp Brain Res 149:439-446. CrossRef Medline

Weiss C, Herwig A, Schütz-Bosbach S (2011) The self in action effects: selective attenuation of self-generated sounds. Cognition 121:207-218. CrossRef Medline

Wolpert DM, Flanagan JR (2001) Motor prediction. Curr Biol 11:R729_ R732. CrossRef Medline

Wolpert DM, Ghahramani Z, Jordan MI (1995) An internal model for sensorimotor integration. Science 269:1880-1882. CrossRef Medline 